\title{
Chronisch entzündliche Darmerkrankungen
}

Chronisch entzündliche Darmerkrankungen (CED) umfassen die Colitis ulcerosa (CU) und den Morbus Crohn (MC). Die Ätiologie beider Erkrankungen ist unbekannt. Pathogenetisch wird ein multifaktorielles Geschehen angenommen in Form einer Reaktion zwischen Darminhalt und Immunsystem der Darmwand, unter Einfluss einer genetischen Suszeptibilität.

Bei CU beginnt die Entzündung im Rektum und breitet sich kontinuierlich oralwärts aus. Der MC unterscheidet sich durch den transmuralen Charakter der Entzündung mit segmentalem Befall, nicht nur des Kolons, sondern potentiell des gesamten Intestinaltraktes. Die Wahl der Behandlung erfordert die Berücksichtigung der individuellen Krankheitssituation.

\section{Therapie der Colitis ullcerosa}

\section{Schub}

Die Behandlung erfolgt bei distaler Lokalisation oral oder rektal, bei (sub-) totalem Befall oral, ggf. auch kombiniert. Medikamente der Wahl sind Aminosalicylate (Sulfasalazin, Mesalazin, Olsalazin) (5) (Abb.1).

Die bevorzugte Behandlung bei distaler CU ist Mesalazin, bei Proktitis mit Suppositorien $(1,0-1,5 \mathrm{~g} / \mathrm{d})$, bei Proktosigmoiditis mit Rektalschäumen $(1 \mathrm{~g} / \mathrm{d})$ oder bei linksseitiger Kolitis mit Klysmen $(2 \mathrm{~g} / \mathrm{d})$. Aminosalicylate sind in der topischen Anwendung den Glucocorticoiden überlegen. Topisch sollten Steroide mit geringer Bioverfügbarkeit verwandt werden (Budesonid, Hydrocortisonacetat). Bei Nichtansprechen einer rektalen Therapie wird mit systemisch wirksamen Glucocorticoiden (oral/ i.v.) begonnen.

Die ausgedehnte CU leichter bis mittlerer Aktivität wird mit oralen Aminosalicylaten behandelt. Im Vergleich zu Sulfasalazin sind Präparate ohne Sulfonamidanteil tendenziell wirkungsvol- ler (7). Im Vergleich findet sich kein eindeutiger therapeutischer Vorteil zwischen Mesalazin und dem Prodrug Olsalazin (3). Die optimale Dosis scheint bei $3 \times 1 \mathrm{~g} / \mathrm{d} 5$-Aminosalicylsäure zu liegen. Bei Nichtansprechen dieser Therapie kann eine kombinierte orale und rektale Gabe von Aminosalicylaten versucht werden.

In der Behandlung der schweren $\mathbf{C U}$ und des toxischen Kolons muss ein chirurgisches Vorgehen frühzeitig erwogen werden. Systemische Glucocorticoide bis zu einer Dosis von $100 \mathrm{mg} / \mathrm{d}$ oral oder im Allgemeinen i.v. sind die Therapie der Wahl (5). Die zusätzliche Gabe von topischen Steroiden per Rektum scheint die Wirksamkeit zu erhöhen.

Auch ohne wissenschaftliche Grundlage werden Antibiotika eingesetzt, z. B. die Kombination Metronidazol und Ciprofloxacin oder alternativ Mezlocillin.

Bestehen Nahrungs- und Flüssigkeitsdefizite, muss i.v. substituiert, gegebenenfalls auch parenteral ernährt werden. Das erhöhte Thromboserisiko bei CU erfordert eine Prävention mit Heparin.

Bei Versagen der intravenösen Behandlung kann eine immunsuppressive Therapie mit Cyclosporin (2 oder $4 \mathrm{mg} / \mathrm{kg}$ pro Tag) (8) eingesetzt werden. Wirkungseintritt kann innerhalb von 710 Tagen erwartet werden. Sobald sich abzeichnet, dass eine Proktokolektomie vermieden werden kann, wird zusätzlich Azathioprin $2 \mathrm{mg} / \mathrm{kg}$ pro Tag gegeben.

\section{Chronisch aktiver Verlauf}

Die Behandlung der chronisch aktiven CU erfordert ein ständiges Abwägen der chirurgischen und konservativen Optionen. Medikamente der Wahl sind Azathioprin 2,0 mg/kg pro Tag und 6-Mercaptopurin $1 \mathrm{mg} / \mathrm{kg}$ pro Tag (5). Azathioprin/6-Mercapto- 


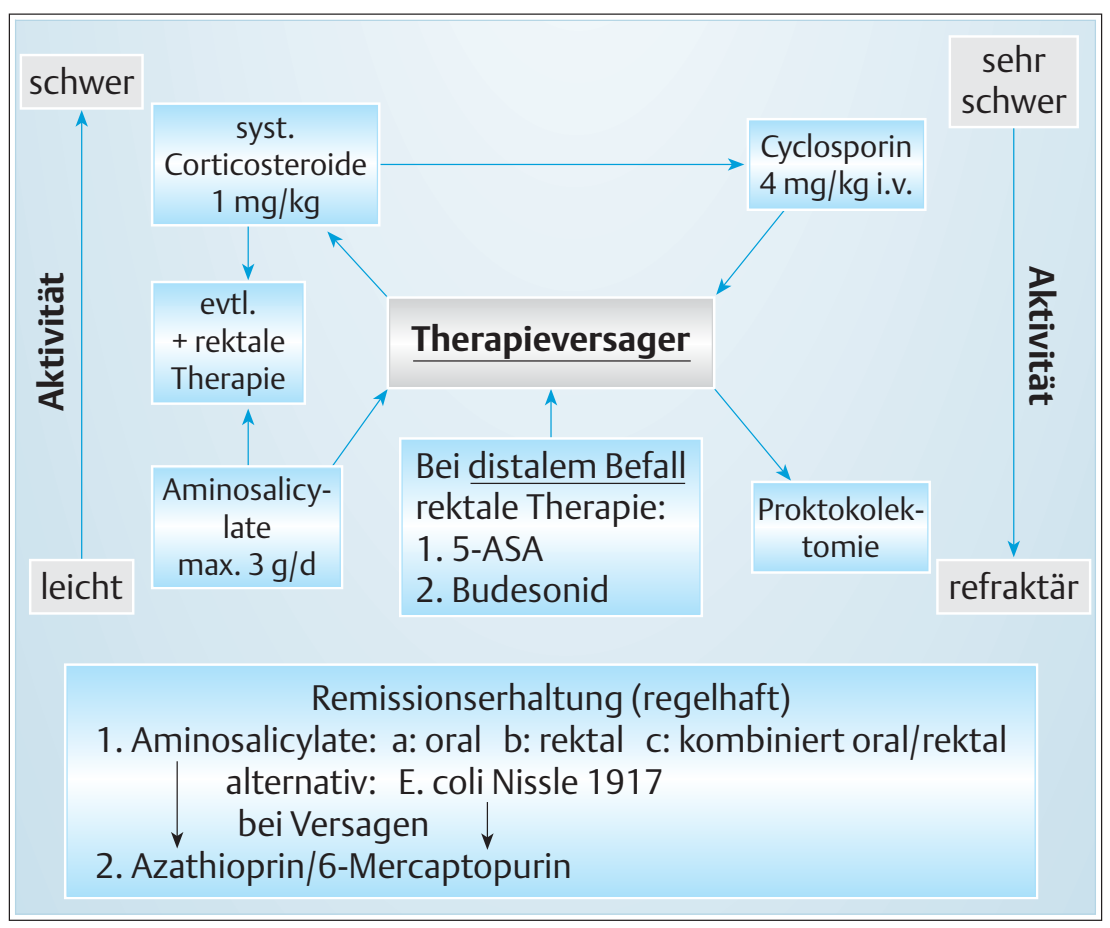

Abb.1 Behandlungsschema für die Colitis ulcerosa.

purin können mit Aminosalicylaten kombiniert werden, wobei sich die Suppression der Granulozytopoese verstärken kann. Eine Dauertherapie mit systemisch wirksamen Glucocorticoiden sollte vermieden werden.

\section{Erhaltung der Remission}

Eine remissionserhaltende Therapie ist streng angezeigt. Sie kann oral und bei entsprechendem Befallsmuster rektal erfolgen (5). Sulfasalazin hat sich im Vergleich zu den anderen Aminosalicylaten als geringfügig überlegen gezeigt (7), allerdings steht diesem Vorteil der Nachteil einer schlechteren Verträglichkeit gegenüber. Dosierungen von 1,5-2,0g/d 5-Aminosalicylsäure werden empfohlen. Eine Kombination aus oraler und rektaler Gabe von Mesalazin ist der alleinigen oralen überlegen.

Die remissionserhaltende Therapie mit dem Probiotikum E. coli Nissle ( $200 \mathrm{mg} / \mathrm{d}$ ) ist wirkungsäquivalent (2). Bei Therapieversagen kann Azathioprin/6-Mercaptopurin in Dosen von 1,4$2,5 \mathrm{mg} / \mathrm{kg}$ pro Tag eingesetzt werden, wobei auf die alternative Möglichkeit einer Proktokolektomie verwiesen wird (Abb.1).

kurzgefasst: Basis der medikamentösen Therapie bei CU sind Aminosalicylate und Corticosteroide, wobei die Besonderheit in der Applikationsform liegt, die neben den üblichen Wegen auch über das Rektum oder kombiniert erfolgen kann.

\section{Therapie des Morbus Crohn}

\section{Schub}

Grundlagen sind die Leitlinien der Deutschen Gesellschaft für Verdauungs- und Stoffwechselerkrankungen (6). Wirksame Medikamente bei mildem bis mäßig aktivem MC sind Mesalazin (3-4g/d) und Budesonid (ileozökaler Befall; $9 \mathrm{mg} / \mathrm{d}$ ), alternativ Prednisolon (-äquivalent) $(30-60 \mathrm{mg} / \mathrm{d})$. Für die unkomplizierte Erkrankung wird bei ileozökalem Befall Budesonid vorge- schlagen und bei Kolonbefall Aminosalicylate (4). Erst bei Versagen dieser Therapie sollte Prednisolon angewandt werden (Abb.2).

Bei schweren Schüben wird initial Prednisolon $(60 \mathrm{mg} / \mathrm{d})$ gegeben, bei häufigen Schüben in der Vorgeschichte in Kombination mit Azathioprin (2-2,5 mg/kg pro Tag) oder 6-Mercaptopurin (1-1,5mg/kg pro Tag). Eine enterale Ernährungstherapie kann bei Ernährungsdefiziten oder Kindern mit Wachstumsstörungen sinnvoll sein.

Antibiotika, Azathioprin/6-Mercaptopurin 2,5 mg/kg pro Tag bzw. $1,5 \mathrm{mg} / \mathrm{kg}$ pro Tag, Methotrexat $25 \mathrm{mg}$ i.m. einmal wöchentlich und Infliximab 5-10 mg/kg KG per infusionem einmalig oder auch wiederholt, sollten in sich steigernder Weise bei therapierefraktären Erkrankungen zum Einsatz kommen.

Bei chronisch-aktivem Krankheitsverlauf wird in erster Linie der langfristige Einsatz von Azathioprin $(2,5 \mathrm{mg} / \mathrm{kg}$ pro Tag) oder 6-Mercaptopurin (1,5 mg/kg pro Tag) empfohlen und in zweiter Linie Methotrexat (15-25mg i.m./Woche). Reservemedikamente sind Infliximab $(5-10 \mathrm{mg} / \mathrm{kg})$ per Infusionen, ggf. Wiederholung alle 8 Wochen.

\section{Remissionserhaltung}

Eine generell durchzuführende Behandlung gibt es nicht, vielmehr ist der individuelle Krankheitsverlauf zu berücksichtigen. Es wird unterschieden zwischen einer medikamentös und einer operativ induzierten Remission (6).

Die Remission wird am besten durch Azathioprin $(2-2,5 \mathrm{mg} / \mathrm{kg}$ pro Tag) bzw. 6-Mercaptopurin (1-1,5mg/kg pro Tag) erhalten. Für Methotrexat (15mg/Woche i.m.) und Infliximab (5-10 mg/ $\mathrm{kg}$ alle 8 Wochen) gibt es eingeschränkt einen Nachweis der remissionserhaltenden Wirksamkeit. Bei der chirurgisch induzierten Remission können Aminosalicylate (3-4g/d) versucht wer- 


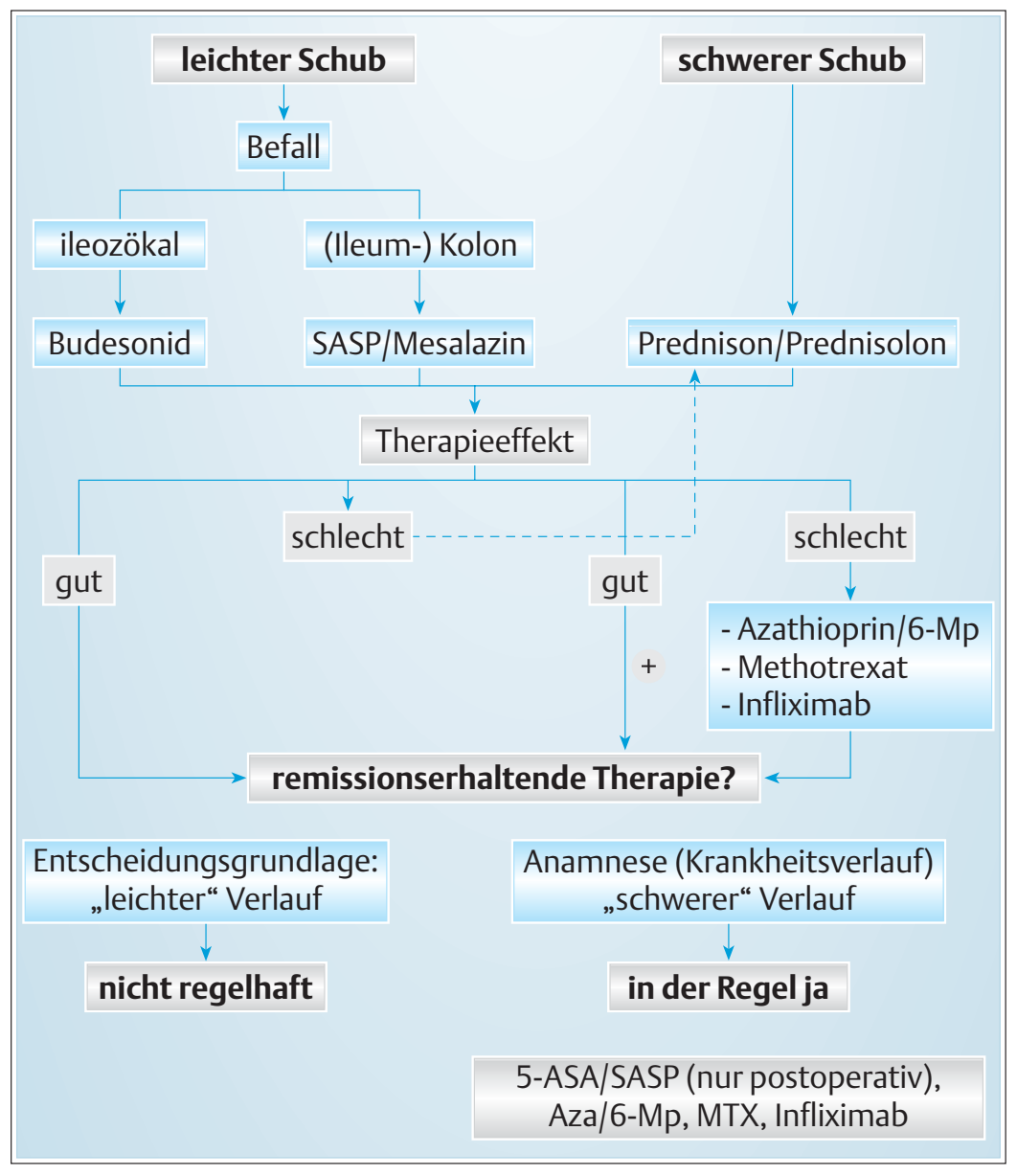

Abb. 2 Behandlungsschema für den Morbus Crohn.

den. Corticosteroide sollten vermieden werden. Nicht rauchen ist wahrscheinlich die wichtigste Empfehlung zur Prognoseverbesserung (1).

kurzgefasst: Neben Aminosalicylaten und Corticosteroiden ist bei MC der rechtzeitige Einsatz von Immunsuppressiva (Azathioprin/6-Mercaptopurin) von besonderer Bedeutung. Operative Alternativen müssen frühzeitig bedacht werden.

\section{Fazit}

Bei der Behandlung von Patienten mit CED sollte einerseits nach den Regeln der evidenzbasierten Medizin vorgegangen werden, andererseits muss die außergewöhnliche Vielfalt der Krankheitsbilder, in der Ausprägung und im Verlauf, Anlass zu einem individualisierten Vorgehen geben. Die grundsätzlich gute Prognose sollte gegen das Inkaufnehmen risikobeladener Behandlungsmöglichkeiten kritisch abgewogen werden. Oft helfen Geduld und der konsequente Einsatz der Standardtherapeutika.

\section{Literatur}

${ }^{1}$ Cosnes J, Beaugerie L, Carbonnel F et al. Smoking Cessation and the course of Crohn's Disease. An intervention study. Gastroenterology 2001; 120: 1093-1099

2 Kruis W, Fric P, Pokrotnieks J et al. Maintaining remission of ulcerative colitis with the probiotic E coli Nissle 1917 is as effective as with standard mesalazine. Gut 2004, in Druck

${ }^{3}$ Kruis W, Pohl C. Klinische Wirkung von verschiedenen 5-ASA-Präparationen bei Colitis ulcerosa. Med Klin 1999; 94 (Suppl 1): 26-29

4 Sandborn WJ, Feagan BG. Review article: mild to moderate Crohn's disease - defining the basis for a new treatment algorithm. Aliment Pharmacol Ther 2003; 18: 263-277

${ }^{5}$ Stange EF, Riemann J, von Herbay A et al. Diagnostik und Therapie der Colitis ulcerosa - Ergebnisse einer evidenz-basierten Konsensuskonferenz der Deutschen Gesellschaft für Verdauungs- und Stoffwechselkrankheiten. Z Gastroenterol 2001; 39: 19-72

${ }^{6}$ Stange EF, Schreiber S, Fölsch UR et al. Diagnostik und Therapie des M. Crohn - Ergebnisse einer evidenzbasierten Konsensuskonferenz der Deutschen Gesellschaft für Verdauungs- und Stoffwechselkrankheiten. Z Gastroenterol 2003; 41: 19-68

7 Sutherland LR, Roth DE, Beck PL. Alternatives to sulfasalazine: a meta-analysis of 5-ASA in the treatment of ulcerative colitis. Inflamm Bowel Dis 1997; 3: 65-78

8 van Assche G, D'Haens G, Noman M et al. Randomized, double-blind comparison of $4 \mathrm{mg} / \mathrm{kg}$ versus $2 \mathrm{mg} / \mathrm{kg}$ intravenous cyclosporine in severe ulcerative colitis. Gastroenterology 2003; 125: 1025-31 